\title{
PET and PET/CT Reports: Observations from the National Oncologic PET Registry
}

\author{
R. Edward Coleman ${ }^{1}$, Bruce E. Hillner ${ }^{2}$, Anthony F. Shields ${ }^{3}$, Fenghai Duan ${ }^{4}$, Denise A. Merlino ${ }^{5}$, Lucy G. Hanna $^{4}$, \\ Sharon Hartson Stine ${ }^{6}$, and Barry A. Siegel ${ }^{7}$ \\ ${ }^{1}$ Department of Radiology, Duke University School of Medicine, Durham, North Carolina; ${ }^{2}$ Department of Internal Medicine and \\ the Massey Cancer Center, Virginia Commonwealth University, Richmond, Virginia; ${ }^{3}$ Karmanos Cancer Institute, Wayne \\ State University, Detroit, Michigan; ${ }^{4}$ Center for Statistical Sciences, Brown University, Providence, Rhode Island; ${ }^{5}$ Society of \\ Nuclear Medicine, Reston, Virginia; ${ }^{6}$ American College of Radiology, Philadelphia, Pennsylvania; and ${ }^{7}$ Division of Nuclear \\ Medicine, Mallinckrodt Institute of Radiology and the Siteman Cancer Center, Washington University School of Medicine, \\ St. Louis, Missouri
}

\begin{abstract}
Our objective was to identify core elements for inclusion in oncologic PET reports and to evaluate a sample of reports in the National Oncologic PET Registry database. Methods: A list of desirable elements in PET reports was compiled from American College of Radiology and Society of Nuclear Medicine guidelines. A training set of 20 randomly selected reports was evaluated by the 4-physician panel, and the results were used to formulate a consensus approach for assessing report content and quality. Each reviewer then scored 65 randomly selected reports-20 common to all reviewers. The scores were tabulated, and interrater variability was measured for the common cases. Results: Each report was assessed for 34 elements-21 primary and 11 additional questions related to 6 of these primary elements. Among the common cases, there was strong $(\geq 0.70)$ interrater agreement for 30 of 34 elements. Among the unique cases, only 9 elements were included in more than $90 \%$ of the reports. Several important elements were not included in more than $40 \%$ of the reports: the reason for the study, a description of treatment history, a statement about comparison to other imaging, and time from radiopharmaceutical injection to imaging. Conclusion: Essential elements that should be included in oncologic PET reports were missing from many reports. These deficiencies may render the reports less helpful to referring physicians, may lead to misdiagnoses, and may cause coding and billing errors. Interpreting physicians should audit their reports to ascertain that they include appropriate elements necessary for billing compliance and for effective communications with referring physicians.
\end{abstract}

Key Words: positron emission tomography; reports; cancer; registry; attributes; coding; billing; management; compliance

J Nucl Med 2010; 51:158-163

DOI: 10.2967/jnumed.109.066399

$\mathbf{T}$ he medical imaging report is the major, and often the only, means of communicating imaging study results from

Received May 20, 2009; revision accepted Jul. 20, 2009.

For correspondence or reprints contact: R. Edward Coleman, Duke University Medical Center, Box 3949, Durham, NC 27710.

E-mail: colem010@mc.duke.edu

COPYRIGHT () 2010 by the Society of Nuclear Medicine, Inc. the interpreting physician to the referring physician. These reports also are used to support the appropriate coding for billing of third-party payers and by payers to determine medical necessity. Accordingly, reports need to contain information that is helpful to referring physicians while describing the rationale for the procedures performed to meet compliance for billing any payer. The American College of Radiology (ACR) has a practice guideline for communication of diagnostic imaging findings (1), and the Society of Nuclear Medicine (SNM) has a section on reporting under its procedure guideline for general imaging (2). These guidelines recommend the elements that should be incorporated in a medical imaging report and describe a suggested format for such reports. Additionally, both the SNM and the ACR have more recent guidelines published in 2006 and 2007, respectively, that list the elements that should be included in reports of PET/CT studies $(3,4)$.

The report elements described in the ACR communication guideline have been used to evaluate the quality of diagnostic radiology reports (5). Reiner and Siegel (6) have noted that, despite the recent major advances in imaging and information technologies, the reports of imaging studies have changed very little. They point out the potential for improved communication through innovations such as speech recognition systems and structured reporting.

The National Oncologic PET Registry (NOPR) is a project conducted under the Center for Medicare and Medicaid Services (CMS) coverage-with-evidence-development program. The NOPR provides coverage for PET and integrated PET/CT (together referred to hereinafter as PET), performed with the glucose analog ${ }^{18} \mathrm{~F}-\mathrm{FDG}$, for previously noncovered cancer types and indications. A description of the NOPR, its design and analysis plan, and its initial results have been published previously (7-11). Briefly, changes in intended management are being evaluated from questionnaire data provided by the referring physician before and after PET. The imaging report is also submitted to the registry database. 
Many radiologists and other physicians interpreting PET studies have had limited training in interpreting and reporting them. Our own anecdotal experiences suggested that there was wide variability in the content and quality of clinical PET reports, but to our knowledge this variability has not been studied systematically. Accordingly, we undertook the present study because of the unique opportunity afforded by the NOPR database to sample PET reports from a broad spectrum of practices throughout the United States. We first prepared a listing of the report elements currently recommended by professional societies and then determined how often those elements were found in the sample of reports studied.

\section{MATERIALS AND METHODS}

The report elements included in our review of PET reports were compiled from the ACR communication guidelines (1), the SNM general imaging guideline (2), and the ACR and SNM PET/CT guidelines $(3,4)$ (Table 1$)$.

A training sample of 20 reports selected randomly from the NOPR database was first reviewed by the 4 physicians on the panel. The reviewers were provided with information from the pre-PET form (8) completed by the referring physician for each case, including the indication for PET, site of proven or suspected cancer (and whether this was pathologically proven), the summary stage, and the pre-PET intended management plan (including treatment type, if that was the plan). Each reviewer graded each report to indicate whether the elements listed in Table 1 were present, not present, or not applicable (if a reviewer judged that a component was appropriately not reported, such as not reporting CT technique if the study was a conventional PET study or not describing the site and intensity of ${ }^{18}$ F-FDG uptake if the study findings were stated to be normal). The results of the initial review of the training set were tabulated and discussed in a conference call to achieve a consensus among the reviewers regarding scoring and to refine the list of elements.

Each reviewer was then sent 65 randomly selected reports. Forty-five of these were unique to each reader, and 20 were common across all readers. The reviewers scored the reports in the same manner as had been done during the training phase. The reviews were submitted on an Excel (Microsoft) spreadsheet to the Brown University Center for Statistical Sciences for analysis.

The ratings for the 20 cases reviewed by all reviewers were analyzed to assess the level of agreement among the reviewers. The frequency of yes responses (element present) was tabulated for each component. The degree of similarity among the readers' responses for each component was assessed by the Rand index, which was computed to measure the proportion of agreement between all possible pairs of reviewers (12). The Rand index has a range of $0-1$, with 0 indicating no similarity between clusters of responses and 1 indicating perfect agreement. The bootstrap method was used to construct the $95 \%$ confidence interval, and a permutation test was conducted to assess whether rater agreement was significantly different from that expected by chance (13).

The results for the 180-case sample made up of the 45 cases unique to each reader were analyzed separately from the 20 cases read by all readers. The frequency and percentage of times each component was present (yes response), as well as the number of times each was not absent (yes or not applicable), were tabulated.

\section{RESULTS}

Three cases were excluded from the analysis, 1 from the common cases and 2 from the set of 180, because the reports either were incomplete or did not appear to correspond to the data on indication and location of cancer. There was generally excellent interrater agreement for the cases read in common by the 4 reviewers. For example, 30 of 34 elements had the agreement $\geq 0.70$ and about half of these had the agreement $\geq 0.90$ across reviewers. On average, the agreement was 0.835 (95\% confidence interval, $0.815-0.853)$ across reviewers and elements. In addition, the level of agreement was significantly different from that expected by chance $(P<0.001)$. There was poorer interrater agreement, as measured by the Rand index, for the following report elements: reason for study clearly stated (range, 0.59 0.90 ), clinical indication for study clearly addressed (range, 0.54-0.72), interpretation of findings provided rather than just restatement of findings (range, 0.52-0.80), and differential diagnosis provided (range, 0.47-0.75).

The characteristics of the reports reviewed individually are noted in Table 2. The sample provided a balanced representation of PET studies: reports from 162 interpreting physicians at 146 PET facilities were included in the sample. Twenty-eight percent of the facilities have hospitalbased scanners, and $25 \%$ have mobile units. The reports span the range of clinical indications: diagnosis, 23.0\%; initial staging, 20.8\%; treatment monitoring, $15.7 \%$; restaging, $18.0 \%$; and detection of recurrence, $22.5 \%$. More than 30 different pathologically proven $(77.5 \%)$ or suspected (12.9\%) cancer types were included: cancers of unknown primary origin and cancers of the pancreas, stomach, and urinary bladder together accounted for $38 \%$. The summary stage of disease was rather evenly distributed. An alternative form of imaging was the most frequent pre-PET management plan, and observation was the least frequent.

The frequency of reporting of each of the report elements is noted in Table 1. Nine elements were included in more than $90 \%$ of the reports for which that element was considered to be applicable: the type or site of cancer; the name of the radiopharmaceutical; the administered activity; the body regions scanned; the date of prior PET or PET/CT studies; the type of prior non-PET studies; the date of prior non-PET studies; the description of the location, extent, and intensity of ${ }^{18} \mathrm{~F}-\mathrm{FDG}$ uptake if an abnormality was present; and a correlation of abnormal PET findings with other results (including the CT findings of a PET/CT study). Some elements that were less commonly included but were considered important were a clear statement of the reason for the study (58\%); a description of treatment history, if applicable (44\%); the date of last treatment (45\%); the blood glucose level (44\%); the time from ${ }^{18} \mathrm{~F}$-FDG injection to imaging (52\%); a characterization of the CT component for PET/CT as nondiagnostic versus diagnostic (54\%); a statement concerning the use of oral contrast material (32\%); a statement concerning the 


\begin{tabular}{|c|c|c|c|c|}
\hline \multirow[b]{2}{*}{ Element } & \multicolumn{3}{|c|}{ Number of reports with element... } & \multirow{2}{*}{$\begin{array}{l}\text { Percentage with } \\
\text { element present, } \\
\text { if applicable }\end{array}$} \\
\hline & Present & Absent & $\begin{array}{l}\text { Not } \\
\text { applicable }\end{array}$ & \\
\hline Reason for study clearly stated?* & 104 & 74 & 0 & 58.4 \\
\hline Type or site of cancer stated, if applicable?* & 154 & 12 & 12 & 92.8 \\
\hline Treatment history described, if applicable?* & 55 & 69 & 54 & 44.4 \\
\hline If yes, date of last treatment given? & 28 & 34 & 116 & 45.2 \\
\hline Radiopharmaceutical name reported? ${ }^{\dagger}$ & 170 & 8 & 0 & 95.5 \\
\hline Administered activity reported? ${ }^{\dagger}$ & 166 & 12 & 0 & 93.3 \\
\hline Route of administration reported? ${ }^{\dagger}$ & 123 & 55 & 0 & 69.1 \\
\hline $\begin{array}{l}\text { Time from injection to imaging } \\
\text { (or time of injection and time of imaging) reported? }\end{array}$ & 92 & 86 & 0 & 51.7 \\
\hline Body region scanned reported? ${ }^{\ddagger}$ & 163 & 15 & 0 & 91.6 \\
\hline CT characterized as nondiagnostic vs. diagnostic? ${ }^{\ddagger}$ & 83 & 72 & 23 & 53.5 \\
\hline Statement indicating whether oral contrast was used?†‡ & 48 & 103 & 27 & 31.8 \\
\hline If yes, type/drug name given? ${ }^{\dagger}$ & 3 & 15 & 160 & 16.7 \\
\hline If yes, dose given ${ }^{\dagger}$ & 4 & 15 & 159 & 21.1 \\
\hline Statement indicating whether IV contrast was used? ${ }^{\dagger \ddagger}$ & 41 & 112 & 25 & 26.8 \\
\hline If yes, type/drug name given ${ }^{\dagger}{ }^{\dagger}$ & 3 & 3 & 172 & 50.0 \\
\hline If yes, dose given?t ${ }^{\dagger}$ & 4 & 1 & 173 & 80.0 \\
\hline $\begin{array}{l}\text { Statement indicating whether comparison was made } \\
\text { with prior PET or PET/CT study (studies) or indicating } \\
\text { that no prior studies are available? }\end{array}$ & 54 & 124 & 0 & 30.3 \\
\hline If yes, type of prior study (studies) identified? & 29 & 7 & 142 & 80.6 \\
\hline If yes, date of prior study (studies) identified? & 34 & 3 & 141 & 91.9 \\
\hline If yes, change from prior study (studies) described? & 27 & 9 & 142 & 75.0 \\
\hline $\begin{array}{l}\text { Statement indicating whether comparison was made } \\
\text { with other prior non-PET imaging study (studies)? }\end{array}$ & 81 & 96 & 1 & 45.8 \\
\hline If yes, type of prior study (studies) identified? & 76 & 0 & 102 & 100.0 \\
\hline If yes, date of prior study (studies) identified? & 72 & 4 & 102 & 94.7 \\
\hline Is the PET study reported as abnormal? ${ }^{*}$ & 136 & 42 & 0 & 76.4 \\
\hline $\begin{array}{l}\text { If yes, location, extent, and intensity of } \\
\text { abnormal }{ }^{18} \mathrm{~F}-\mathrm{FDG} \text { uptake described?* }\end{array}$ & 131 & 4 & 43 & 97.0 \\
\hline SUV or SUVs reported? & 97 & 37 & 44 & 72.4 \\
\hline $\begin{array}{l}\text { Abnormal PET findings correlated with other imaging } \\
\text { results, including concurrent } \mathrm{CT} \text { for } \mathrm{PET} / \mathrm{CT} \text { ? }\end{array}$ & 124 & 13 & 41 & 90.5 \\
\hline Incidental CT findings described (for PET/CT)? & 99 & 58 & 21 & 63.1 \\
\hline Clinical indication for study clearly addressed? & 99 & 79 & 0 & 55.6 \\
\hline $\begin{array}{l}\text { Interpretation of findings provided rather than } \\
\text { just restatement of findings? }\end{array}$ & 145 & 22 & 11 & 86.8 \\
\hline Differential diagnosis provided? & 48 & 110 & 20 & 30.4 \\
\hline Need for follow-up or additional studies described? & 51 & 126 & 1 & 28.8 \\
\hline $\begin{array}{l}\text { Documentation of communication of study findings } \\
\text { to referring MD (or surrogate)? }\end{array}$ & 4 & 174 & 0 & 2.2 \\
\hline Blood glucose reported? $¥ \S$ & 79 & 99 & 0 & 44.4 \\
\hline
\end{tabular}

\footnotetext{
*Element required under coding system of World Health Organization, which has designated National Center for Health Statistics as responsible for coordinating disease classification in United States (describes diagnosis, which in turn supports medical necessity or reason why test was performed).

†Element required under coding system of CMS for Healthcare Common Procedure Coding System level II codes (radiopharmaceuticals and supplies).

${ }^{\ddagger}$ Element required under coding system of American Medical Association for Current Procedural Terminology level I, II, and III codes (level I describes procedure performed).

${ }^{\S}$ Element required under coding system of CMS for clinical laboratory improvement amendments (regulates all laboratory testing, except research, performed on humans in United States).
}

use of intravenous contrast material (27\%); documentation of comparison with a prior scan or that none was available (30\%); and documentation that the study's clinical indication was clearly addressed (56\%).

\section{DISCUSSION}

The content of the report of an imaging test has important ramifications for how a patient is managed and the study reimbursed. If elements related to the performance of 
TABLE 2. Characteristics of Cases Reviewed Individually

Characteristics of cases reviewed individually*

Facilities $(n=146)$

Number of facilities

$n$

146

Hospital- or non-hospital-based

scanner $(n=146)$

PET scanner not hospital-based

PET scanner hospital-based

Mobile or fixed PET scanner $(n=146)$

Stationary

Mobile

Cases reviewed

Number of interpreting physicians

Integrated PET/CT $(n=178)$

PET/CT

PET

Indication $(n=178)$

Diagnosis

Initial staging

Monitoring treatment response

Restaging

Suspected recurrence

Location of cancer $(n=178)$

Pancreas

Unknown primary

Stomach

Bladder

Ovary and uterine adnexa

Kidney and other urinary tract

Prostate

Liver and intrahepatic bile ducts

Lung, non-small cell

Lung, small cell

Other cancer

Cancer confirmation $(n=178)$

Pathologically proven

Clinically suspected

Unknown primary cancer

Summary stage $(n=178)$

No evidence of disease

Localized only

Regional by direct extension, lymph node

Metastatic with a single suspected site

Metastatic with multiple suspected sites

Unknown or uncertain

Pre-PET management plan $(n=178)$

Observation

Additional imaging

Tissue biopsy

Curative treatment

Palliative treatment

*Does not include cases read by all reviewers. submitted by the referring physicians on the pre-PET and post-PET forms, in order to check for the internal consistency of submitted data. During this review, it became apparent to us that many elements that were considered important in a report were not being included. We then undertook this review of a random sample of reports submitted to NOPR to document how often the various recommended report elements were and were not included. We found, confirming our initial impression, that many important elements of a PET report were missing in a large fraction of reports, including such items as a clear statement of the reason for the study and a description of previous treatment history. Important technical details often missing or omitted were the blood glucose level, the time from radiopharmaceutical injection to imaging, and the details of the CT technique in PET/CT studies. Several elements may have been available to the interpreting physician and that information may have been used in the interpretation of the study (we could not determine this from the available data), but if these are not documented in the report, information that is potentially important to the referring physician may be lost. Moreover, if the elements are not included in the report, there is no documentation that they were available to the interpreting physician.

Some elements that were included in the review are expected to be in all reports, if applicable, and others are not. For example, all reports should contain the following elements: the reason for the study; type or site of cancer (if applicable); treatment history (if applicable); type of therapy (surgery, radiation therapy, or chemotherapy) and timing of therapy relative to the PET scan; radiopharmaceutical, route of administration, and administered activity; blood glucose level; time from injection to imaging; body region scanned; whether a diagnostic $\mathrm{CT}$ scan was performed and, if it was, the type and amount of oral and intravenous contrast material used; whether comparison was made with prior imaging studies; location, extent, and intensity of abnormality (including standardized uptake value, if appropriate); correlation with findings of other imaging studies; incidental CT findings for PET/CT; clinical indication clearly addressed; interpretation of the findings in the report impression (rather than just a restatement of findings); differential diagnosis (if appropriate); and documentation of communication of study findings to referring physician (if appropriate). Some interpreting physicians may not consider each of these elements essential to include in the report. For example, some may not report the type and amount of contrast material used since it is included in their facility's procedure protocol. In general, we consider it important to include this information for comparison of techniques when 2 scans are performed at different facilities. Reports of PET studies done for diagnosis would not be expected to specify the type of cancer and treatment history but should specify the reason cancer is suspected clinically. Not all reports need a differential diagnosis. For example, if a patient has biopsy-proven lung cancer, the scan is done for staging, and 
no other abnormalities are detected, a differential diagnosis is not needed. Unexpected or urgent findings on the PET scan and incidental findings on the CT scan that may alter management of the patient should be communicated to the referring physician or surrogate. The need to communicate is dependent on the findings and clinical context. For example, in a patient undergoing an initial staging scan for lung cancer, an adrenal metastasis is not an unexpected finding (but may need to be communicated if the patient is known to be scheduled for surgery the next day), but a previously unknown large lytic metastasis of the femoral neck seen on PET and CT images would certainly warrant communication to the referring physician.

Several factors, particularly having the clinical history available (14) and performing a comparison with previous radiologic studies $(15,16)$ at the time of image interpretation, have been reported to increase the accuracy of the interpretations. In a recent review of diagnostic accuracy, Berlin (17) notes that having clinical information and reviewing previous studies decreases the number of misdiagnoses. More accurate reports would be expected to be more helpful to referring physicians and ultimately lead to better patient management. Documenting in a report that clinical information was considered and comparison with prior studies was performed informs the referring physician of key factors considered by the reader.

There are some important aspects of the report that relate and directly link to coding, billing, and compliance $(5,18)$. Payer and coding guidelines state that if the nature of a procedure is not documented in the report, one cannot bill for that procedure. Title XVIII of the Social Security Act, section 1862 (a) (1) (A), allows coverage and payment for services provided to Medicare patients considered medically reasonable and necessary; section 1833 (e) prohibits Medicare payment for any claim that lacks the necessary documentation (in this case, in the PET report) to support the charges on the claim. At a minimum, reporting codes that are not clearly documented in the report could be considered fraudulent if audited for regulatory compliance $(5,18)$.

For PET procedures, CMS has issued specific medical necessity requirements in a series of national coverage determinations, the most recent of which was published on April 3, 2009 (19). The new framework for Medicare coverage of oncologic PET categorizes studies as either for initial treatment strategy (formerly diagnosis and initial staging) or subsequent treatment strategy (formerly treatment monitoring and restaging); later this year, new modifiers will need to be appended to the procedure code to indicate the purpose of the study. The national coverage determination imposes a frequency limit on studies done for initial treatment strategy and requires that some PET studies still must be done under the CMS coverage-withevidence-development program. An appropriately detailed history that addresses the CMS-defined criteria for initial treatment and subsequent treatment strategies is necessary to determine whether the procedure meets medical neces- sity for the code billed. Additionally, CMS considers surveillance analogous to screening and not coverable; therefore, it is prudent to document the rationale for PET requested to detect a suspected recurrence in order to demonstrate the medical necessity of the study as a condition of coverage. Lack of detailed information could result in an inappropriate choice of code or modifiers.

Because Medicare has contractual obligations to its beneficiaries and the Medicare Trust Fund, the CMS, as well as other payers, requires reasonable, complete, and legible documentation that services are consistent with coverage policies. Documentation includes clinical descriptions in the report that are consistent with definitions contained in Current Procedural Terminology, International Classification of Diseases and Healthcare Common Procedure Coding System codes and past and present diagnoses that should be accessible and accurately reported to the highest degree of certainty. In Table 1 we have noted the report elements that are necessary for accurate and appropriate coding.

There are several reasons some of the elements may not be in a report. Some of the information that ideally should be included in the report may not have been available to the interpreting physician at the time of dictation (e.g., blood glucose level, time from injection to imaging, and the reason for the study). Frequently a request will just include the type of cancer, but the physician interpreting the study does not know the specific reason for the study. Such a situation suggests a need for the PET center to revise its request initiation process and the types of information the requesting physician should be expected to provide.

\section{CONCLUSION}

Several elements that should be in a PET report are not present in most reports. Only 9 of the 34 elements reviewed were found in more than $90 \%$ of the reports. These deficiencies may result in reports that are less helpful to referring physicians, cause misdiagnoses, and lead to coding and billing errors. Interpreting physicians should audit their reports to ascertain that they include appropriate elements necessary for billing compliance and for effective communications with referring physicians.

\section{ACKNOWLEDGMENT}

Funding for development of the National Oncologic PET Registry was provided by the Academy for Molecular Imaging, but the registry is otherwise self-supported by the fees paid by participating PET facilities.

\section{REFERENCES}

1. American College of Radiology. ACR practice guideline for communication of diagnostic imaging findings. 2005. Available at: http://www.acr.org/Secondary MainMenuCategories/quality_safety/guidelines/dx/comm_diag_rad.aspx. Accessed September 29, 2009. 
2. Parker JA, Daube-Witherspoon ME, Graham LS, et al. Society of Nuclear Medicine procedure guideline for general imaging. Version 3.0. 2004. Available at: http://interactive.snm.org/docs/General_Imaging_v3.0.pdf. Accessed September $29,2009$.

3. American College of Radiology. ACR practice guideline for performing FDGPET/CT in oncology. 2007. Available at: http://www.acr.org/SecondaryMain MenuCategories/quality_safety/guidelines/nuc_med/fdg_pet_ct.aspx. Accessed September 29, 2009.

4. Delbeke D, Coleman RE, Guiberteau MJ, et al. Procedure guideline for tumor imaging with ${ }^{18}$ F-FDG PET/CT 1.0. 2006. Available at: http://interactive.snm. org/docs/jnm30551_online.pdf. Accessed September 29, 2009.

5. Weiner SN. Radiology by non-radiologists: is report documentation adequate? Am J Manag Care. 2005;11:781-785.

6. Reiner B, Siegel E. Radiology reporting; returning to our image-centric roots. AJR. 2006;157:1151-1155.

7. Lindsay MJ, Siegel B, Tunis SR, et al. The National Oncologic PET Registry: expanded Medicare coverage for PET under coverage with evidence development. AJR. 2007;188:1109-1113.

8. Hillner BE, Siegel BA, Coleman RE, et al. The National Oncologic PET Registry (NOPR): design and analysis plan. J Nucl Med. 2007;48:19011908.

9. Hillner BE, Siegel BA, Liu D, et al. The impact of positron emission tomography/computed tomography (PET/CT) and PET on expected management of patients with cancer: initial results from the National Oncologic PET Registry. J Clin Oncol. 2008;26:2155-2161.
10. Hillner BE, Siegel BA, Shields AF, et al. The impact of positron emission tomography on expected management during cancer treatment: findings of the National Oncologic PET Registry. Cancer. 2009;115:410-418.

11. Hillner BE, Siegel BA, Shields AF, et al. Relationship between cancer type and the impact of PET and PET/CT on intended management: findings of the National Oncologic PET Registry. J Nucl Med. 2008;49:1928-1935.

12. Rand WM. Objective criteria for the evaluation of clustering methods. J Am Stat Assoc. 1971;66:846-850.

13. Efron B, Tibshirani RJ. An Introduction to the Bootstrap. New York, NY: Chapman and Hall; 1993.

14. Loy CT, Irwig L. Accuracy of diagnostic tests read with and without clinical information: a systematic review. JAMA. 2004;292:1602-1609.

15. Doubilet $P$, Herman PG. Interpretation of radiographs: effect of clinical history. AJR. 1981;137:1055-1058.

16. White K, Berbaum K, Smith WL. The role of previous radiographs and reports in the interpretation of current radiographs. Invest Radiol. 1994;29:263-265.

17. Berlin L. Accuracy of diagnostic procedures: has it improved over the past five decades? AJR. 2007;188:1173-1178.

18. Thorwarth WT Jr. Get paid for what you do: dictation patterns and impact on billing accuracy. J Am Coll Radiol. 2005;2:665-669.

19. Centers for Medicare and Medicaid Services. Decision memo for positron emission tomography (FDG) for solid tumors (CAG-00181R). 2009. Available at: http:// www.cms.hhs.gov/mcd/viewdecisionmemo.asp?id=218. Accessed September 29, 2009. 